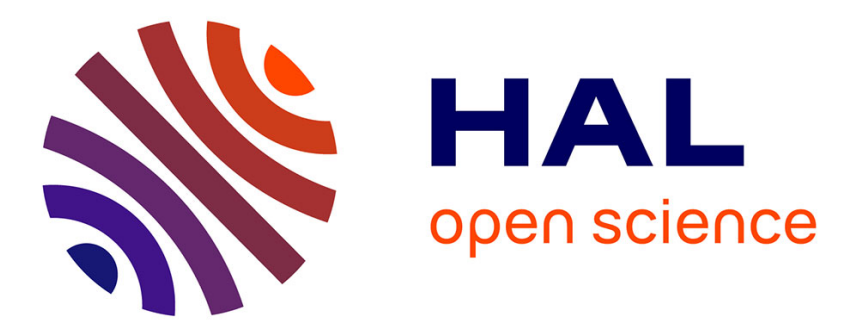

\title{
Irradiance Dependence of the Short-Circuit Current Temperature Coefficient of sc-Si PV Module
}

\author{
Baojie Li, Anne Migan-Dubois, Claude Delpha, Demba Diallo
}

\section{To cite this version:}

Baojie Li, Anne Migan-Dubois, Claude Delpha, Demba Diallo. Irradiance Dependence of the ShortCircuit Current Temperature Coefficient of sc-Si PV Module. IEEE Photovoltaic Specialists Conference (PVSC 2020), Jun 2020, Calgari (virtual), Canada. 10.1109/PVSC45281.2020.9300416 . hal02903351

\section{HAL Id: hal-02903351 \\ https://hal-centralesupelec.archives-ouvertes.fr/hal-02903351}

Submitted on 4 Nov 2021

HAL is a multi-disciplinary open access archive for the deposit and dissemination of scientific research documents, whether they are published or not. The documents may come from teaching and research institutions in France or abroad, or from public or private research centers.
L'archive ouverte pluridisciplinaire HAL, est destinée au dépôt et à la diffusion de documents scientifiques de niveau recherche, publiés ou non, émanant des établissements d'enseignement et de recherche français ou étrangers, des laboratoires publics ou privés. 


\title{
Irradiance Dependence of the Short-Circuit Current Temperature Coefficient of sc-Si PV Module
}

\author{
Baojie LI \\ GeePs - Université Paris-Saclay, CentraleSupélec, CNRS \\ L2S - Université Paris-Saclay, CNRS, CentraleSupélec \\ Gif-sur-Yvette, France \\ baojie.li@centralesupelec.fr \\ Anne MIGAN-DUBOIS \\ GeePs - Université Paris-Saclay, CentraleSupélec, CNRS \\ Gif-sur-Yvette, France \\ anne.migan-dubois@geeps.centralesupelec.fr
}

\author{
Claude DELPHA \\ L2S - Université Paris-Saclay, \\ CNRS, CentraleSupélec \\ Gif-sur-Yvette, France \\ claude.delpha@centralesupelec.fr
}

\author{
Demba DIALLO \\ GeePs - Université Paris-Saclay, \\ CentraleSupélec, CNRS \\ Gif-sur-Yvette, France \\ demba.diallo@ centralesupelec.fr
}

\begin{abstract}
Temperature coefficient of short-circuit current $\left(T C_{I_{S C}}\right)$ of $\mathrm{PV}$ modules plays an important role in $\mathrm{PV}$ modeling and I-V characteristic correction. Many standards and researches have indicated that $T C_{I_{S C}}$ is irradiance-dependent, but the detailed relationship is not really clarified. Thus, this paper investigates the relationship of module $T C_{I_{S C}}$ with irradiance from both indoor and outdoor tests and reveal the impact of the varying $T C_{I_{S C}}$ in common applications.
\end{abstract}

Keywords-Photovoltaics, temperature coefficient, short-circuit current, current correction, sc-Si module

\section{INTRODUCTION}

Temperature coefficient of short-circuit current $\left(T C_{I_{S C}}\right)$ of photovoltaic (PV) module is a crucial parameter for PV system [1]. It is widely used for PV modeling [2] and the correction of field-measured current data [3], e.g., the current at maximum power point $\left(I_{m p p}\right)$ or the current of I-V curve. The application in current correction, specifically, is also an important step for various subsequent performance and reliability analysis, such as PV degradation assessment [4], PV fault detection and diagnosis [5].

Generally, $T C_{I_{S C}}$ is provided in the manufacture datasheet but always for Standard Test Condition (STC, module temperature $T_{m}=25^{\circ} \mathrm{C}$, in-plane irradiance $G=1000 \mathrm{~W} / \mathrm{m}^{2}$, air mass $\mathrm{AM}=1.5)$. However, many standards like $[3,6]$ have pointed out that the value of $T C_{I_{S C}}$ is only valid within a certain range of irradiance. Nevertheless, there is little research dedicated to explain how the module $T C_{I_{S C}}$ varies with $G$. In the literature, Muhammad et al. [7] observed a decreasing trend of
$T C_{I_{S C}}$ with $G$, but the research is limited to cell level. Manoj et al. [8] focused on the module level $T C_{I_{S C}}$ and captured similar trend, but the precision is hindered as there are only 4 observations measured at different $T_{m}$ for each $G$, which are inadequate to fully exhibit the relationship of $I_{S C}$ with $T_{m}$ and to extract reliable $T C_{I_{S C}}$ values.

Since the dependence of $T C_{I_{S C}}$ on $G$ is not always clear, therefore, in most cases, it is considered as a constant value for convenience $[4,5]$. However, the impact of this assumption is rarely specified. Consequently, this paper is conceived to evaluate the relationship of module $T C_{I_{S C}}$ with $G$ by both indoor and outdoor tests and then discuss the impact of the varying $T C_{I_{S C}}$ in its common applications.

\section{Methodology of $T C_{I_{S C}}$ CALCUlation}

According to IEC 60891 [3], the calculation of module $T C_{I_{S C}}$ can be summarized in the following steps:

- Step 1: Collect module $I_{S C}$ measured at same $G$ level but with different $T_{m}$;

- Step 2: Plot $I_{S C}$ as a function of $T_{m}$, fit the whole curve linearly with a least-squares function;

- Step 3: Record the slope of the fitted line as the absolute $T C_{I_{S C}}$ (expressed in $\mathrm{A} /{ }^{\circ} \mathrm{C}$ ), while the relative (expressed in $\% /{ }^{\circ} \mathrm{C}$ ) could be determined by dividing the absolute coefficient with the $I_{S C}$ value at STC.

In this calculation procedure, Step 1 is relatively difficult because of its two simultaneous constraints on $G$ and $T_{m}$. 
Besides, a continuous variation of $T_{m}$ in a wide range $\left(\geq 30^{\circ} \mathrm{C}\right)$ is also suggested in [3]. Some test schemes can be adopted to collect the required $I_{S C}$. Using solar simulator and $T_{m}$ control device is one choice, which permits to perform superior spectrum match. However, the expense of this test is also high, especially when dealing with PV samples of large size, e.g., PV module sample instead of cell sample. Consequently, two cost efficient test procedures are proposed in the followings and adopted in our research to collect the required $I_{S C}$ for the study of $T C_{I_{S C}}$.

- Procedure 1: Collect data in indoor controlled irradiance condition (e.g., using Xenon or Halogen lamps)

- Procedure 2: Collect data under natural sunlight in a short time (assuming $G$ varies very little in this interval)

The details and the experimental setup of these two test procedures will be presented in the next section.

\section{EXPERIMENTAL SETUP}

\section{A. Basic experimental test bench}

A MX-50Wp sc-Si module is considered for both indoor and outdoor tests with the datasheet parameters shown in TABLE I.

TABLE I. PARAMETERS OF TESTED SC-SI MODULE

\begin{tabular}{|c|c|c|c|}
\hline Parameters & Value & Parameters & Value \\
\hline$V_{O C}$ & $21.9 \mathrm{~V}$ & $I_{S C}$ & $2.97 \mathrm{~A}$ \\
\hline$V_{M P}$ & $18.6 \mathrm{~V}$ & $V_{M P}$ & $2.68 \mathrm{~A}$ \\
\hline Relative $T C_{V_{O C}}$ & $0.28 \% /{ }^{\circ} \mathrm{C}$ & Relative $T C_{I_{S C}}$ & $0.074 \% /{ }^{\circ} \mathrm{C}$ \\
\hline
\end{tabular}

A reference cell (RG100) measures the plane-of array irradiance $\left(G_{P O A}\right)$ and a Pt100 probe (class A) measures the back-sheet $T_{m}$. The module $I_{S C}$ is measured with a programmed source meter (Keithley 2440).

\section{B. Indoor test under controlled irradiance}

The test setup is shown in Fig. 1, where 4 halogen lamps provide illumination (non-uniformity $<10 \%$ ) to the tested module. $G$ could be adjusted continuously from 0 to 1400 $W / m^{2}$ by controlling the lamp current. The specific test procedure contains the following steps:

- Step 1: Cool down the test module by using an electric fan with the lamps turned off until the backsheet $T_{m}$ stabilizes to room temperature.

- Step 2: Adjust $G$, let the module warm up by the effect of illumination until the backsheet $T_{m}$ reaches stable operating temperature. During the process, record $G_{P O A}$, $T_{m}$ and $I_{S C}$ every 2 seconds.

- Step 3: Repeat Step 1-2 with different $G$ values.
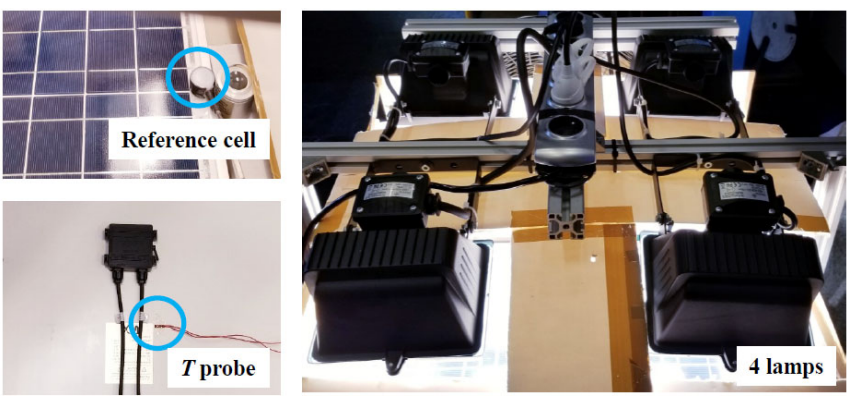

Fig. 1. Experimental setup of indoor controlled -irradiance test

\section{Outdoor short-time test under natural sunlight}

Outdoor test has a similar test procedure:

- Step 1: Cool the module with a fan in shaded place until $T_{m}$ stabilized to ambient temperature.

- Step 2: In cloudless weather, move the module (with measurement devices together installed on a movable trolley as shown in Fig. 2) to sunlit place.

- Step 3: Let the module naturally warm up in short time (minimize the impact of changing $G$ ) with $T_{m}$ approaching operating temperature. During the process, record $G_{P O A}, T_{m}$ and $I_{S C}$ every 2 seconds.

- Step 4: Repeat Step 1-3 for different time slots in the same day for different $G$ levels.

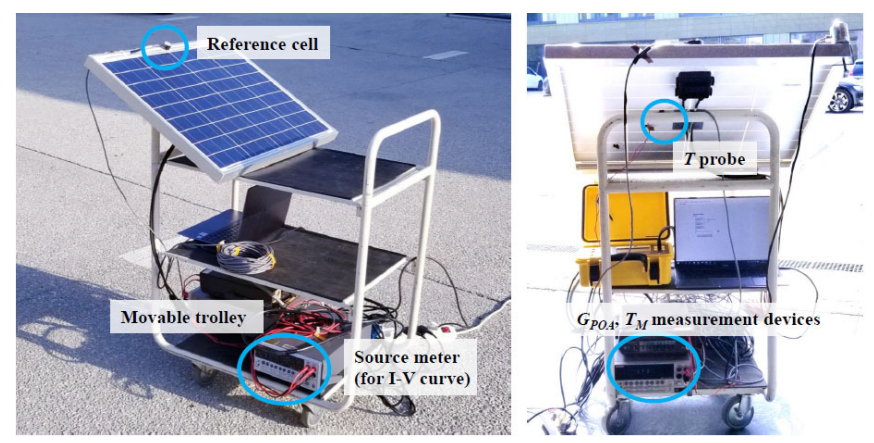

Fig. 2. Experimental setup of outdoor short-time test

\section{RESULTS AND ANALYSIS}

\section{A. Correction of $I_{S C}$ based on measured $G_{P O A}$}

In fact, based on our measurement results, $G$ is not stable for both indoor and outdoor tests, i.e., indoor $G_{P O A}$ varies by $\pm 3 \%$, due to voltage fluctuation while outdoor $G_{P O A}$ varies by $\pm 4 \%$, due to the change of sun position). Thus, it is necessary to correct the $I_{S C}$ measured under variable $G$ to a value $\left(I_{S C}^{C}\right)$ under same $G$ (here, $G$ at STC) via the following equation [3]:

$$
I_{S C}^{c}=I_{S C} \cdot G_{S T C} / G
$$

After the correction, $T C_{I_{S C}}$ could then be calculated based on the procedure presented in Section II.

\section{B. Indoor test results}

Several indoor tests were performed with a $G_{P O A}$ covering a range from 300 to $1100 \mathrm{~W} / \mathrm{m}^{2}$. One test result with an average 
$G_{P O A}$ at $883 \mathrm{~W} / \mathrm{m}^{2}$ is presented in Fig. 3 to illustrate the calculation of $T C_{I_{S C}}$.

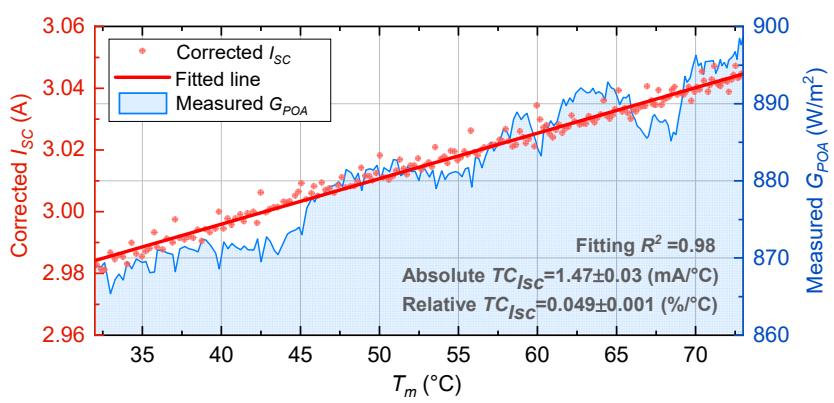

Fig. 3. Indoor test result with average $G_{P O A}$ at $883 \mathrm{~W} / \mathrm{m}^{2}$

As illustrated in Fig. 3, the corrected $I_{S C}$ exhibits a linear relationship with $T_{m}$ during the test period ( $T_{m}$ varies from 32 to $73^{\circ} \mathrm{C}$ ). After linear fitting via Matlab (fitting $R^{2}=0.98$ ), the absolute and the relative $T C_{I_{S C}}$ (dividing the absolute value by $I_{S C}$ at $\mathrm{STC}$ from datasheet) are obtained as $1.47 \mathrm{~mA} /{ }^{\circ} \mathrm{C}$ and $0.049 \% /{ }^{\circ} \mathrm{C}$, respectively. In this manner, the relative $T C_{I_{S C}}$ of all the tests can be obtained.The results are presented in Fig. 4 the corresponding variation of $T_{m}$ also illustrated.

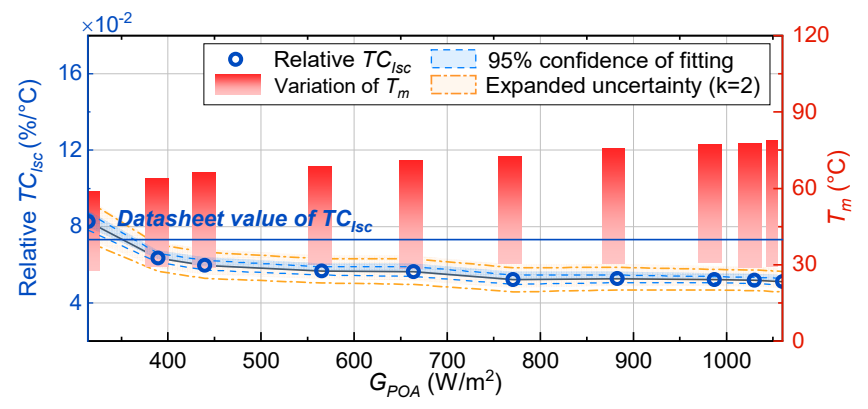

Fig. 4. Indoor test results under different irradiance levels

It is observed from that Fig. 4, firstly, for each test, the $T_{m}$ of tested module, via warming up by the effect of illumination, spreads a wide range of variation. The difference between inital and final $T_{m}$ could reach or exceed $30^{\circ} \mathrm{C}$, especially for the test at high $G_{P O A}$. This wide range of variation permits to fully examine the linear relationship of $I_{S C}$ with $T_{m}$ and extract the $T C_{I_{S C}}$ with more precision.

As for the behavior of $T C_{I_{S C}}$, the relative $T C_{I_{S C}}$ shows a decreasing trend with $G_{P O A}$ and the decline rate slows down as $G_{P O A}$ increases. Therefore, $T C_{I_{S C}}$ at high $G_{P O A}\left(G_{P O A}>700\right.$ $\left.W / m^{2}\right)$ is relatively stable, but the value is inferior to the datasheet value with the relative error up to $31.1 \%$.

\section{Outdoor test results}

From 8 am to 12 am on 2019/09/03, several outdoor tests were performed. Taking one test result (shown in Fig. 5) as example, a linear evolution of corrected $I_{S C}$ with $T_{m}$ is also observed but with larger fluctuations (fitting $R^{2}=0.78$ ) than the indoor test result. This may be due to the more variable environmental conditions (e.g., impact of wind).

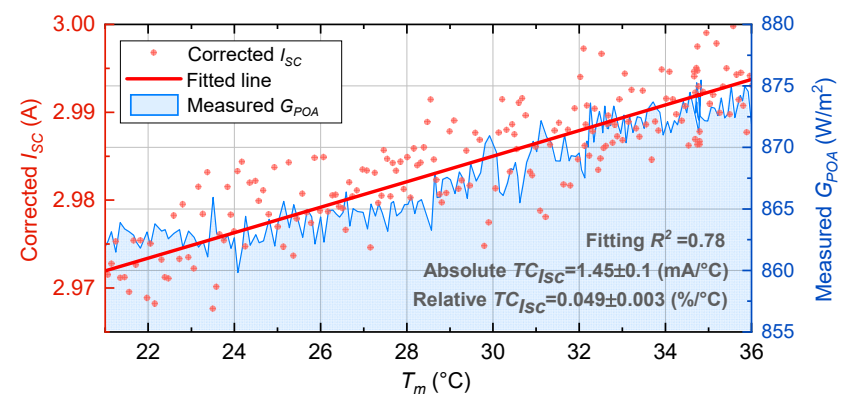

Fig. 5. Outdoor test result with average $G_{P O A}$ at $868 \mathrm{~W} / \mathrm{m}^{2}$

Similarly, the calculated $T C_{I_{S C}}$ at different sunlight are plotted in Fig. 6. From the results, firstly, it is observed that the variation of $T_{m}$ increases gradually with $G_{P O A}$ and but is relatively lower than that for indoor test. This is mainly due to the fact that outdoor tests are carried on in open space (where ambient temperature is relatively stable during each short-time test) rather than the confined space of indoor tests (where the ambient temperature also increases), so the variation of $T_{m}$ is not that significant.

Regarding the $T C_{I_{S C}}$, a similar decreasing trend of $T C_{I_{S C}}$ with $G_{P O A}$ is also found. There are certain parts not perfectly matching with the indoor test results, like the $T C_{I_{S C}}$ at low $G_{P O A}$ range, where larger values are observed. Nevertheless, the general variation tendency is coherent with that of indoor test results, like decreasing decline rate with $G_{P O A}$ and the relatively stabilized value at high $G_{P O A}$ range. In this stabilized range, the value of $T C_{I_{S C}}$ is found also coherent, which are around $0.05 \% /{ }^{\circ} \mathrm{C}$.

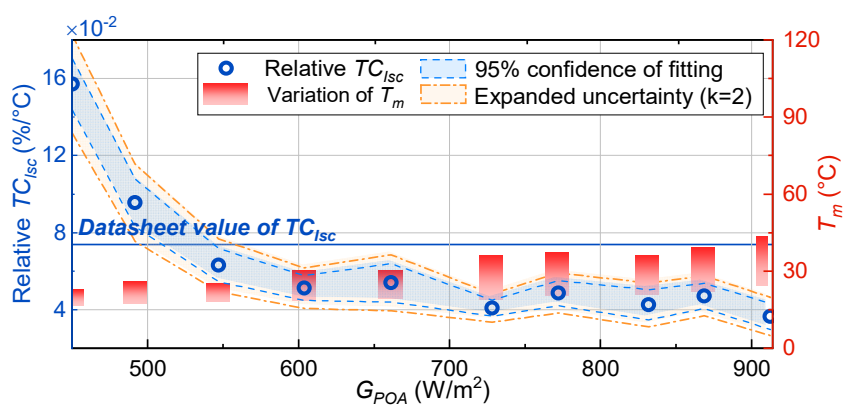

Fig. 6. Outdoor test results under sunlight irradiance levels

\section{Effect of a Variable $T C_{I_{S C}}$}

From indoor and outdoor tests, the calculated $T C_{I_{S C}}$ shows significant variation particularly with the $G_{P O A}$ at low values $\left(<400 \mathrm{~W} / \mathrm{m}^{2}\right)$. In order to evaluate the impact of this variation, let's consider the application in current correction using (2) as an example.

$$
I_{2}=I_{1} \cdot\left(1+T C_{I_{S C}} \cdot\left(T_{2}-T_{1}\right)\right) \cdot G_{2} / G_{1}
$$

where, $I_{1}$ and $I_{2}$ are the current before and after correction; $G_{1}$ and $G_{2}, T_{1}$ and $T_{2}$ are the measured and desired irradiance or module temperature, respectively. 
Assuming that $I_{1}$ is the measured current at low irradiance $G_{1}$ (e.g., $400 \mathrm{~W} / \mathrm{m}^{2}$ ) and the measured $T C_{I_{S C}}$ at $G_{1}$ is $0.16 \% /{ }^{\circ} \mathrm{C}$ (according to Fig. 6). When $\left(T_{2}-T_{1}\right)$ drops in the range $\left[20^{\circ} \mathrm{C}, 50^{\circ} \mathrm{C}\right]$, and if the datasheet value of $T C_{I_{S C}}\left(0.07 \% /{ }^{\circ} \mathrm{C}\right)$ is used to correct $I_{1}$, the relative error of $I_{2}$, compared to the result using the real measured $T C_{I_{S C}}$, lies between 1.7-4.2\%. If using $T C_{I_{S C}}$ at high $G_{P O A}\left(\right.$ e.g., $\left.0.05 \% /{ }^{\circ} \mathrm{C}\right)$, the error will reach $2.1-$ $5.1 \%$. These errors can hinder the realization of high-precision current correction and degrade the current-based module performance analysis.

\section{UNCERTAINTY ANALYSIS}

The uncertainty characterizes the range of values within which the true value of one measurand is considered to lie with certain level of confidence [9]. The uncertainty involved in the calculation of $T C_{I_{S C}}$ is determined by the uncertainty of 3 measurands, i.e., $G_{P O A}, T_{m}$ and $I_{S C}$. For each measurand, the uncertainty addresses the measurement error from 2 effects, the random effect caused by the stochastic variation of measurement device and the systematic effect caused by persistent issue which leads to a consistent error [10]. For $G_{P O A}$, the systematic effect mainly results from the spectral mismatch of reference cell, the alignment of sensor with tested module [11], etc.; For $T_{m}$, the systematic effect is mainly caused by the calibration, uniformity of backsheet $T_{m}$ and the difference between the rear side $T_{m}$ and the cell temperature [11]. Using infrared (IR) camera, the distribution of module $T_{m}$ is available to examine. Two examples taken during the outdoor tests are shown in Fig. 7. It is observed a clear nonuniform distribution of $T_{m}$, which is commonly encountered due to the wind and physical irregularities (like module frame and junction box) [12]. The maximum variation of rear side $T_{m}$ is within $3^{\circ} \mathrm{C}$, which meets the requirement proposed in [3] for the correction analysis.
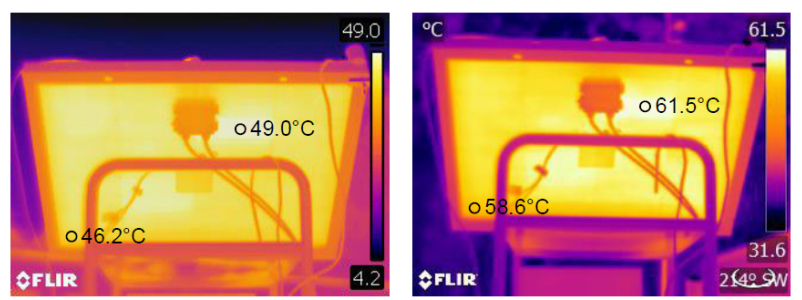

Fig. 7. IR images of module backside during outdoor tests

In order to quantify the impact of these uncertainty on $T C_{I_{S C}}$, the uncertainty propagation analysis is performed. Firstly, since relative $T C_{I_{S C}}$ is calculated by the slope of $I_{S C}^{c}$ with $T_{m}$ (by linear fitting) normalized by $I_{S C}^{S T C}$, a simplified function, which is based on the difference of $T_{m}\left(\Delta T_{m}\right)$ and $I_{S C}^{c}\left(\Delta I_{S C}^{c}\right)$ between 2 observations (with similar $G$ but considerably different $T_{m}$ ), is used to present the calculation of the relative $T C_{I_{S C}}$ as:

$$
T C_{I_{S C}}=\frac{1}{I_{S C}^{S T C}} \cdot \frac{\Delta I_{S C}^{c}}{\Delta T_{m}}
$$

Then, replace the $\Delta I_{S C}^{c}$ by the difference of the corresponding measured $I_{S C}\left(\Delta I_{S C}\right)$ via (1) (supposing the $G$ of the 2 observations are identical):

$$
T C_{I_{S C}}=\frac{1}{I_{S C}^{S T C}} \cdot \frac{\Delta I_{S C} \cdot \frac{G_{S T C}}{G}}{\Delta T_{m}}=\frac{G_{S T C}}{I_{S C}^{S T C}} \cdot \frac{\Delta I_{S C}}{\Delta T_{m} G}
$$

In this manner, the relation of relative $T C_{I_{S C}}$ with the measured parameters, $T C_{I_{S C}}=f\left(G, \Delta I_{S C}, \Delta T_{m}\right)$, is available for uncertainty analysis. Here, we consider the relative standard uncertainty $\left(u^{r}\right)$, which is the absolute uncertainty divided by the best estimate of one measurand [9]. For a function $y=$ $f\left(X_{1}, X_{2}, X_{3}\right)=K \cdot X_{1} /\left(X_{2} X_{3}\right)$, where $K$ is a constant and $X_{i}(i=1,2,3)$ are the measurands, the propagated $u_{y}^{r}$ based on the $u_{X_{i}}^{r}(i=1,2,3)$ could be calculated as [9]:

$$
\left|u_{y}^{r}\right|=\sqrt{\sum_{i=1}^{3} u_{X_{i}}^{r 2}+2 \sum_{i=1}^{2} \sum_{j=i+1}^{3} u_{X_{i}}^{r} u_{X_{j}}^{r} r\left(X_{i}, X_{j}\right)}
$$

where $r\left(X_{i}, X_{j}\right)$ represents the correlation coefficient between $X_{i}$ and $X_{j}$.

Based on the datasheet of measurement devices and the reported common measurement systematic uncertainty $[11,13]$, the combined $u_{G}^{r}, u_{\Delta I_{S C}}^{r}$ and $u_{\Delta T}^{r}$ are considered as $5 \%, 0.1 \%$ and $2 \%$ (using a coverage factor $k=2$ ), respectively. Here, the $u^{r}$ of $\Delta I_{S C}$ and $\Delta T_{m}$ are set relatively lower than that of $G$. This is out of the consideration that the parameters $\left(\Delta I_{S C}\right.$ and $\left.\Delta T_{m}\right)$ used in (4) are the difference values, thus the impact of systematic error (like calibration bias, the difference between rear side $T_{m}$ and cell $T$ ), which is generally larger than the random dispersion, could be reduced.

In fact, all the measurands $G, \Delta I_{S C}$ and $\Delta T_{m}$ are correlated but with different $r$. For example, $\Delta I_{S C}$ is quasi linearly correlated with $G$ with $r\left(G, \Delta I_{S C}\right) \approx 1$, while $\Delta T_{m}$, i.e., the variation of the $T_{m}$ between 2 chosen observations, is nearly uncorrelated with $G$ or $\Delta I_{S C}$. Therefore, for simplification, we consider $r\left(G, \Delta I_{S C}\right)=1, r\left(\Delta T_{m}, \Delta I_{S C}\right)=r\left(\Delta T_{m}, G\right)=0$. In this way, based on (5), the propagated $u^{r}$ of $T C_{I_{S C}}$ could be expressed as:

$$
\left|u_{T C_{I S C}}^{r}\right|=\sqrt{u_{G}^{r 2}+u_{\Delta I_{S C}}^{r 2}+u_{\Delta T}^{r 2}+2 \cdot u_{G}^{r} \cdot u_{\Delta I_{S C}}^{r}}
$$

The $u_{T C_{I S C}}^{r}$ equals $5.5 \%$, which means that the $T C_{I_{S C}}$ will fluctuate by $\pm 5.5 \%$ around the calculated value due to the uncertainty of the 3 measurands. Now, we add this uncertainty to the results obtained in previous section and reshow them in Fig. 8 and Fig. 9. From the results, it is observed that the uncertainty introduced by the 3 measurands has no significant impact on the global decreasing trend of the obtained $T C_{I_{S C}}$. 


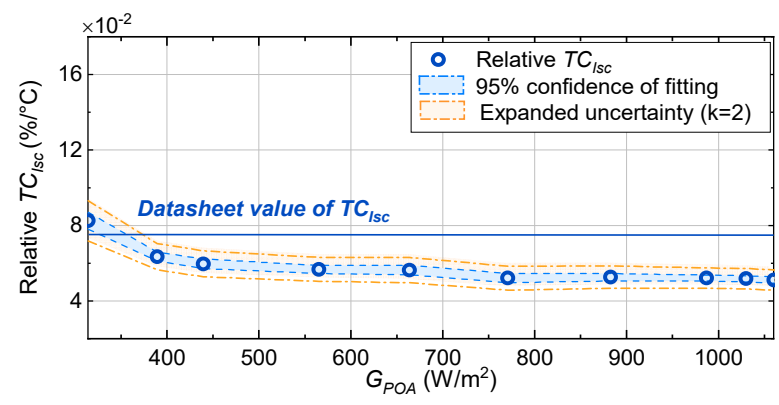

Fig. 8. Indoor test results with uncertainty added

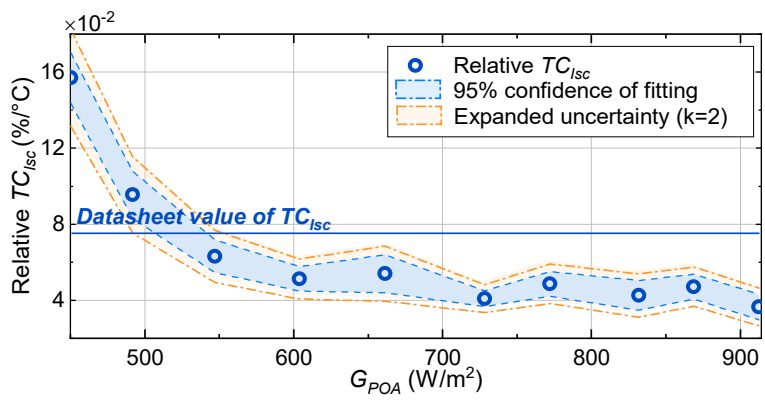

Fig. 9. Outdoor test results with uncertainty added

\section{DISCUSSION}

The observed trend in the variation of $T C_{I_{S C}}$ with $G$ is consistent with the reported results at cell level [7] and at module level under solar simulator [8]. Since $T C_{I_{S C}}$ has been validated spectrum-dependent [14], this $G$-dependent phenomenon may be partially the impact of spectrum. For outdoor test under clear sky, from morning to noon, $G$ increases with time, but the energy portion in near infrared range decreases. Since $T C_{I_{S C}}$ responds significantly to this range of wavelength [15], the change of spectrum could lead to the decrease of $T C_{I_{S C}}$. However, for indoor test, the spectrum is relatively stable. Therefore, this could not fully explain the phenomenon. It clearly requires further exploration.

\section{CONCLUSION}

The dependence of sc-Si module $T C_{I_{S C}}$ on irradiance is investigated in this paper. A decreasing trend is observed from both indoor and outdoor tests. The decline rate slows down as $G_{P O A}$ increases. Thus $T C_{I S C}$ at high $G_{P O A}$ range is almost constant but to a value lower than the one given in datasheet. We have shown that if an improper value of $T C_{I S C}$ is applied, an error of up to $5 \%$ in current correction could be reached. Future research will continue to study the $G$ dependence of $T C_{I S C}$ on other technologies of PV modules (like a-Si, CdTe, HIT), to quantify the impact of several factors (like $I_{S C}$ correction method, installation position of $T_{m}$ probe) on the test results and to analyze the underlying mechanism of the variation of $T C_{I_{S C}}$.

\section{REFERENCES}

[1] R Dubey, P Batra, S Chattopadhyay, A Kottantharayil, BM Arora, KL Narasimhan, et al. "Measurement of temperature coefficient of photovoltaic modules in field and comparison with laboratory measurements," in Proceedings of IEEE 42nd Photovoltaic Specialist Conference, New Orleans, LA, USA, 14-17 Jun., 2015, pp. 1-5.

[2] I de la Parra, M Muñoz, E Lorenzo, M García, J Marcos, F MartínezMoreno. "PV performance modelling: A review in the light of quality assurance for large PV plants," Renew Sustain Energy Rev, vol. 78, pp. 780-97, 2017.

[3] IEC 60891. "Photovoltaic devices - Procedures for temperature and irradiance corrections to measured I-V characteristics," IEC, Standard, 2009

[4] J Eduardo, FS De Oliveira, C Wilhelm, M Prieb. "Degradation analysis of a photovoltaic generator after operating for 15 years in southern Brazil," Sol Energy, vol. 196, no. 2019, pp. 196-206, 2020.

[5] H Zhu, L Lu, J Yao, S Dai, Y Hu. "Fault diagnosis approach for photovoltaic arrays based on unsupervised sample clustering and probabilistic neural network model," Sol Energy, vol. 176, pp. 395-405, 2018.

[6] IEC 61853-1. "Photovoltaic (PV) module performance testing and energy rating - Part 1: Irradiance and temperature performance measurements and power rating," IEC, Standard, 2011

[7] T Muhammad, O Jan Ove, S Tor Oskar. "Temperature Coefficients of Solar Cells from Elkem Solar under Varied Intensities and Selective Wavelength Regions of the $\backslash$ textsc $\{$ AM $\} 1.5$ Spectrum," in Proceedings of 29th EU PVSEC 2014, Amsterdam, Netherlands, 22-24 Sep., 2014, pp. 3378-82.

[8] M Morampudi, B Bora, G Kumar, R Kumar, R Siddiqui, S et al. "Performance comparision of PV module based of temperature cofficient in indoor and outdoor conditions as per IEC 61853-1," in Proceedings of 32nd EU PVSEC 2016, Munich, Germany, 21-24 Jun., 2016, pp. 2067-9.

[9] JCGM. "Evaluation of measurement data-Guide to the expression of uncertainty in measurement Évaluation des données de mesure-Guide pour l'expression de l'incertitude de mesure," JCGM, Report, 2008

[10] JM Bland, DG Altman. "Statistics notes: Measurement error," BMJ, vol. 312, no. 7047, pp. 1654-1654, 1996.

[11] C Reise, B Müller, D Moser, G Belluardo, P Ingenhoven. "Report IEAPVPS T13-12:2018 Uncertainties in PV System Yield Predictions and Assessments," IEA, Report, 2018

[12] DL King, JA Kratochvil, WE Boyson. "Temperature coefficients for PV modules and arrays: Measurement methods, difficulties, and results," in Proceedings of the 26th IEEE Photovoltaic Specialists Conference, Anaheim, CA, USA, 29 Sept.-3 Oct., 1997, pp. 1183-6.

[13] D Dirnberger, U Kraling. "Uncertainty in PV module measurement-part i: Calibration of crystalline and thin-film modules," IEEE J Photovoltaics, vol. 3, no. 3, pp. 1016-26, 2013.

[14] CR Osterwald, M Campanelli, T Moriarty, KA Emery, R Williams. "Temperature-dependent spectral mismatch corrections," IEEE J Photovoltaics, vol. 5, no. 6, pp. 1692-7, 2015.

[15] K Ramspeck, F Plag, F Haas, S Winter, A Metz. "Temperature Coefficients of Silicon Solar Cells," in Proceedings of 31st EU PVSEC 2015, Hamburg, Germany, 14-18 Sep., 2015, pp. 799-803. 